Edith Cowan University

Research Online

ECU Publications Post 2013

$1-1-2014$

\title{
Simultaneous monitoring of singlet and triplet exciton variations in solid organic semiconductors driven by an external static magnetic field
}

\author{
Baofu Ding \\ Edith Cowan University \\ Kamal Alameh \\ Edith Cowan University
}

Follow this and additional works at: https://ro.ecu.edu.au/ecuworkspost2013

Part of the Other Mechanical Engineering Commons

10.1063/1.4890315

This is an Author's Accepted Manuscript of: Ding B., Alameh K. (2014). Simultaneous monitoring of singlet and triplet exciton variations in solid organic semiconductors driven by an external static magnetic field. Applied Physics Letters, 105(1). Available here

This Journal Article is posted at Research Online.

https://ro.ecu.edu.au/ecuworkspost2013/332 


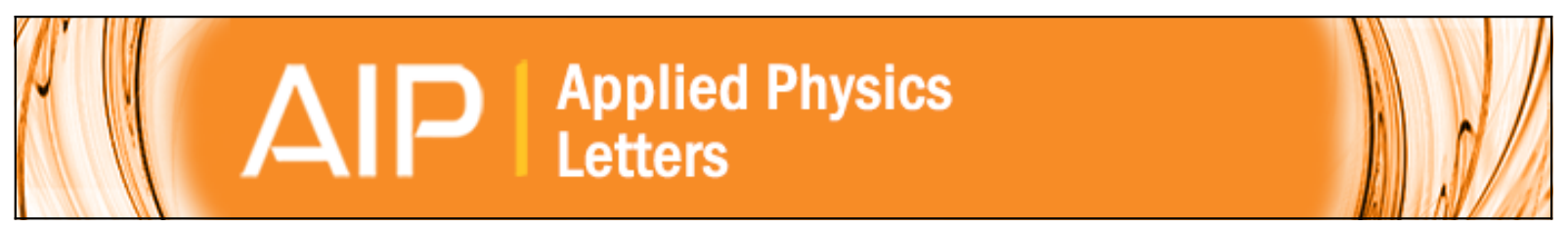

\section{Simultaneous monitoring of singlet and triplet exciton variations in solid organic semiconductors driven by an external static magnetic field}

Baofu Ding and Kamal Alameh

Citation: Applied Physics Letters 105, 013304 (2014); doi: 10.1063/1.4890315

View online: http://dx.doi.org/10.1063/1.4890315

View Table of Contents: http://scitation.aip.org/content/aip/journal/apl/105/1?ver=pdfcov

Published by the AIP Publishing

\section{Articles you may be interested in}

Nanoscopic mechanisms of singlet fission in amorphous molecular solid

Appl. Phys. Lett. 102, 173301 (2013); 10.1063/1.4795138

Causes of efficiency roll-off in phosphorescent organic light emitting devices: Triplet-triplet annihilation versus triplet-polaron quenching

Appl. Phys. Lett. 97, 243304 (2010); 10.1063/1.3527085

Dissociation processes of singlet and triplet excitons in organic photovoltaic cells

Appl. Phys. Lett. 89, 131116 (2006); 10.1063/1.2357584

Effects of ferromagnetic nanowires on singlet and triplet exciton fractions in fluorescent and phosphorescent organic semiconductors

Appl. Phys. Lett. 88, 022114 (2006); 10.1063/1.2162801

High-efficiency yellow double-doped organic light-emitting devices based on phosphor-sensitized fluorescence Appl. Phys. Lett. 79, 1045 (2001); 10.1063/1.1388159

\section{A|P| $\left.\right|_{\text {Applied Physics }} ^{\text {Journal of }}$}

Journal of Applied Physics is pleased to announce André Anders as its new Editor-in-Chief 


\title{
Simultaneous monitoring of singlet and triplet exciton variations in solid organic semiconductors driven by an external static magnetic field
}

\author{
Baofu Ding ${ }^{\text {a) }}$ and Kamal Alameh ${ }^{\text {b) }}$ \\ Electron Science Research Institute, Edith Cowan University, 270 Joondalup Drive, Joondalup, WA 6027, \\ Australia
}

(Received 11 April 2014; accepted 3 July 2014; published online 11 July 2014)

\begin{abstract}
The research field of organic spintronics has remarkably and rapidly become a promising research area for delivering a range of high-performance devices, such as magnetic-field sensors, spin valves, and magnetically modulated organic light emitting devices (OLEDs). Plenty of microscopic physical and chemical models based on exciton or charge interactions have been proposed to explain organic magneto-optoelectronic phenomena. However, the simultaneous observation of singlet- and triplet-exciton variations in an external magnetic field is still unfeasible, preventing a thorough theoretical description of the spin dynamics in organic semiconductors. Here, we show that we can simultaneously observe variations of singlet excitons and triplet excitons in an external magnetic field, by designing an OLED structure employing a singlet-exciton filtering and detection layer in conjunction with a separate triplet-exciton detection layer. This OLED structure enables the observation of a Lorentzian and a non-Lorentzian line-shape magnetoresponse for singlet excitons and triplet excitons, respectively. (C) 2014 AIP Publishing LLC.
\end{abstract}

[http://dx.doi.org/10.1063/1.4890315]

During the past two decades, organic semiconductors have been investigated intensively for the development of organic light emitting devices (OLEDs), ${ }^{1-3}$ organic photovoltaic cells (OPVs), ${ }^{4}$ and organic transistors (OTs). ${ }^{5}$ Generally, organic semiconductors have weak spin-orbit interaction, hence, they possess long spin-relaxation time. ${ }^{6}$ Such feature has triggered a promising research field, namely, organic spintronics, ${ }^{7-14}$ which has attracted great interest due to its potential applications in displays, data storage, environmental sensors, and photovoltaics. Recently, it has experimentally been observed that an external magnetic field affects the singlet and triplet exciton ratio in organic semiconductors, and the exploitation of such spintronic effects could bring enormous benefits to the fields of OLEDs, OPVs, and OTs. ${ }^{15-18}$ For example, applying the small magnetic field could enable the large amplification of either singlet or triplet excitons, leading to a significant increase in the efficiency of the electroluminescence in OLEDs. While many proposed microscopic physical models, based on spin exchange, spin scattering, and spin mixing, are conceptually straightforward, the simultaneous monitoring of singlet and triplet exciton variations in solid organic semiconductors driven by an external static magnetic field has not been achieved experimentally. ${ }^{10,13,19,20}$ This is simply because the relaxation of an excited triplet to a singlet ground state of different spin symmetry is forbidden. Therefore, the triplet excitons do not contribute to the magnetoelectroluminescence (MEL) in conventional OLEDs. To investigate the impact of an external magnetic field on both the fluorescence and phosphorescence of OLEDs, Reufer et al. have proposed an approach based on embedding phosphorescent sensitizers into organic semiconductors and measuring the

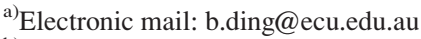

${ }^{\text {b)} E l e c t r o n i c ~ m a i l: ~ k . a l a m e h @ e c u . e d u . a u ~}$
}

electroluminescence spectra for different external magnetic fields. ${ }^{21}$ However, the internal energy transfer between singlet and triplet excitons makes such an approach inaccurate for the discrimination of singlet-exciton variation from triplet-exciton variation, because both singlet and triplet excitons contribute to electrophosphorescence. ${ }^{22}$ So far, the observation of triplet-exciton variation in an external magnetic field is still a challenging topic.

In the present work, we propose an approach, based on the use of singlet-exciton filtering and detection layer and a triplet-exciton detection layer in order to block the internal energy transfer between singlet excitons and triplet excitons, as illustrated in Fig. 1. This approach enables simultaneous monitoring of singlet and triplet exciton variations in an external magnetic field. Two proof-of-concept OLED structures are developed, and the experimental results confirm that the relationship governing the singlet exciton variation versus magnetic field is a Lorentzian line-shape, and show that the triplet exciton variation with the magnetic field is a quasi-linear non-Lorentzian line-shape.

The device fabrication processes are described as follows: 220-nm indium tin oxide (ITO)-coated glass substrates were cleaned in detergent, acetone, and ethanol alternately, and followed by ultraviolet (UV)-Ozone-treatment. The substrates were then transferred to a high vacuum chamber. The organic semiconducting materials used here include Tris-(8hydroxyquinoline) aluminum $\left(\mathrm{Alq}_{3}\right), \mathrm{N}, \mathrm{N}^{\prime}$-bis(l-naphthyl)$\mathrm{N}, \mathrm{N}^{\prime}$-diphenyl-1,1'-biphentl-4,4'-diamine (NPB), Coumarin 540 (C540), and 2,3,7,8,12,13,17,18-octaethyl-21H,23H-porphine platinum(II) (PtOEP). The film thicknesses were realtime detected by using a quartz crystal thickness monitor. The metal electrodes were also prepared by vacuum thermal deposition. All the organic materials and Al were deposited by a thermal evaporation method in a vacuum of $10^{-6} \mathrm{~Pa}$. A $40 \mathrm{~nm}$ thick film of the hole transporting material, NPB, was 


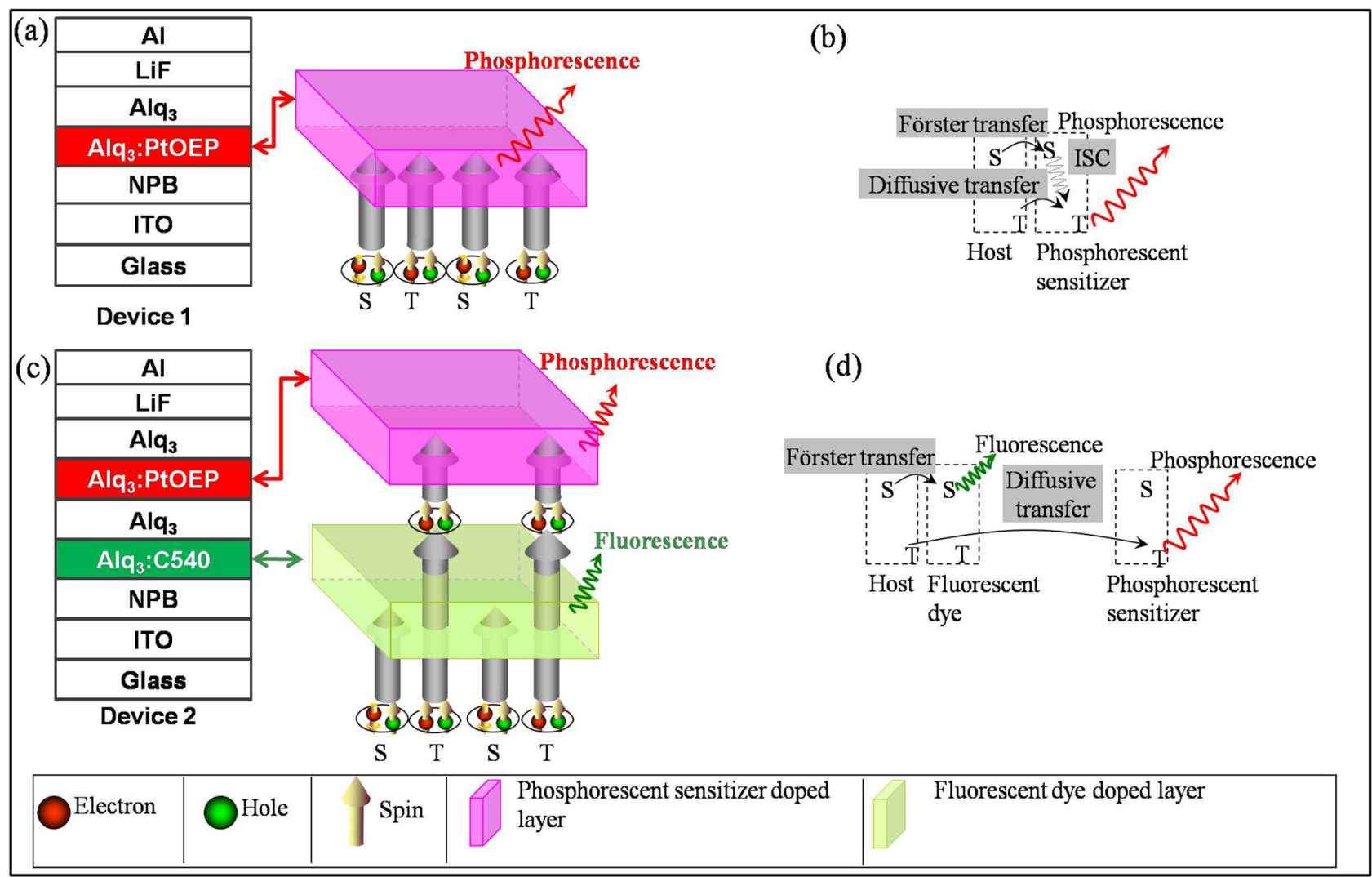

FIG. 1. Schematic view of the light emission from ((a) and (b)) conventional phosphorescent OLED and ((c) and (d)) the proposed OLED with a singletexciton filtering and detection layer and a triplet-exciton detection layer.

evaporated onto the ITO coated glass substrates. After that, a $90 \mathrm{~nm}$ thick $\mathrm{Alq}_{3}$ for electron transporting and emitting layer, $8 \AA$ thick $\mathrm{LiF}$ for electron-injection improvement, and a $100 \mathrm{~nm}$ thick $\mathrm{Al}$ for a cathode were successively deposited. The emitting area was $4 \times 4 \mathrm{~mm}^{2}$. The doped layers in device 1 and device 2 were fabricated through co-evaporating the host material and the dye material. The doping ratios were controlled by tuning their evaporating rates. The fabricated devices were then encapsulated in a $\mathrm{N}_{2}$ glove box with oxygen and water content less than $1 \mathrm{ppm}$. Spectra of electroluminescence were measured using a PR705 spectrometer. An electromagnet, generating a magnetic field of up to $200 \mathrm{mT}$, was used for investigating the effect of magnetic-field on exciton formation. The magneto-fluorescence and magnetophosphorescence were measured by placing the developed OLEDs in a magnetic field generated by the electromagnet. The PR705 spectrometer was about $1.5 \mathrm{~m}$ away from the electromagnet, ensuring its sensitivity to the wavelength at this distance was not affected by the electromagnet. The observed exciton formation was found to be independent of the orientation of the magnetic field with respect to the OLEDs.

Fig. 2 shows the magneto-phosphorescence of device 1: ITO/NPB $(40 \mathrm{~nm}) / \mathrm{PtOEP}$ doped $\mathrm{Alq}_{3}(10 \mathrm{~nm}) / \mathrm{Alq}_{3}(80 \mathrm{~nm}) /$ $\mathrm{LiF}(0.8 \mathrm{~nm}) / \mathrm{Al}$. $\mathrm{Alq}_{3}$ and $\mathrm{NPB}$ were, respectively, chosen as the electron-transporting and light-emitting layer and the holetransporting layer, and an aluminum film was chosen as the cathode. To increase the electron injection efficiency, a thin layer of $\mathrm{LiF}$ buffer was inserted between the $\mathrm{Al}$ and $\mathrm{Alq}_{3}$ layers, as typically done in conventional OLEDs. PtOEP was used as phosphorescent dye in $\mathrm{Alq}_{3}$ hosts with a volume concentrating ratio of $6 \%$. Magneto-phosphorescence or magneto- fluorescence is defined as $\Delta \mathrm{Ph} / \mathrm{Ph}=(\mathrm{Ph}(\mathrm{B})-\mathrm{Ph}(0)) / \mathrm{Ph}(0)$ or $\Delta \mathrm{Fl} / \mathrm{Fl}=(\mathrm{Fl}(\mathrm{B})-\mathrm{Fl}(0)) / \mathrm{Fl}(0)$, where $\mathrm{Ph}$ and $\mathrm{Fl}$ denote the electro-phosphorescence and electro-fluorescence, respectively, and $\mathrm{Ph}(\mathrm{B})(\mathrm{Fl}(\mathrm{B}))$ and $\mathrm{Ph}(0)(\mathrm{Fl}(0))$ are the electrophosphorescence (electro-fluorescence) with and without an external magnetic field of B, respectively. As shown in Fig. 2, for magnetic fields $<25 \mathrm{mT}$, fast increase in magnetophosphorescence is displayed, whereas for higher magnetic fields, the increase in magneto-phosphorescence is quasi-linear with a small slope.

As shown in Figs. 1(a) and 1(b), there are two kinds of internal energy transfer between the host molecules and phosphorescent sensitizers in device 1, namely, a conventional phosphorescent-sensitizer-doped OLED. ${ }^{6}$ One is long-

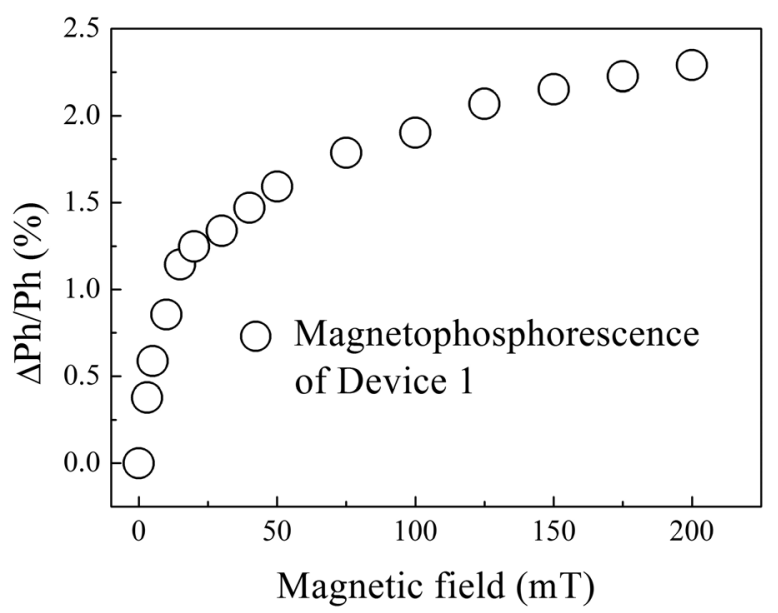

FIG. 2. Magneto-phosphorescence response of device 1. 
range Förster energy transfer occurring between singlet states, and the other one is short-range Dexter transfer occurring between the triplet states. Generally, in material chemistry, the phosphorescent sensitizer has heavy metal elements, such as Pt and Ir. The heavy metal elements introduce strong spin-orbit coupling which not only reduces the phosphorescence life time but also enhances the efficiency of intersystem crossing (ISC) from singlet excited states to triplet excited states. Consequently, both singlet excitons and triplet excitons in host molecules can excite triplet excitons in sensitizers as illustrated in Fig. 1(b). Therefore, as discussed in Ref. 22, the variation of phosphorescence in an external magnetic field cannot purely reflect the triplet-exciton variation in the host material, because besides the triplet excitons, the singlet excitons from the host molecules also contribute to the phosphorescence emitted by the phosphorescent sensitizers. As discussed above, both singlet excitons and triplet excitons in the host material contribute to the electro-phosphorescence emitted by the phosphorescent sensitizer through Förster energy transfer, Dexter Transfer, and ISC. Therefore, it is inferable that the magneto-phosphorescence in Fig. 2 consists of two components, namely, singlet-exciton variations and triplet-exciton variations. It is easy to detect the response of singlet excitons to external magnetic fields, since most of host organic semiconductors are fluorescent materials.

To distinguish triplet-exciton variation from singletexciton variation, an OLED structure (device 2), shown in Fig. 1(c), is proposed and developed, and its energy diagram is shown in Fig. 1(d). The OLED structure has three important features, namely, (1) it has a singlet-filtering layer in front of the phosphorescent-dye-doped layer; (2) the distance between the two doped layers is longer than the characteristic length of Förster energy transfer; (3) the fluorescent dye is appropriately selected to ensure that the electroluminescence spectra from the two doped layers do not overlap. The $1^{\text {st }}$ and $2^{\text {nd }}$ features ensure that the internal energy transfer between singlet excitons and triplet excitons in the fluorescent-dye-doped layer is avoided. ${ }^{6}$ As illustrated in Figs. 1(c) and $1(\mathrm{~d})$, the phosphorescence is mainly due to triplet excitons that diffuse from the singlet-filtering layer to the phosphorescent-dye-doped layer. Consequently, the fluorescentdye-doped layer (S-Layer) is responsible for singlet filtering and detection whereas the phosphorescent-dye-doped layer (T-Layer) performs triplet detection. Note that the $3^{\text {rd }}$ feature ensures that the changes in fluorescent and phosphorescent spectra are independent, thus the variations of singlet excitons and triplet excitons can be measured simultaneously. C540 doped $\mathrm{Alq}_{3}$ and PtOEP doped $\mathrm{Alq}_{3}$ were chosen as the $\mathrm{S}$-Layer and T-Layer, respectively. The spacing between the $\mathrm{S}$-Layer and T-Layer was $20 \mathrm{~nm}$, which is much longer than the characteristic 8-nm radius of Förster energy transfer in $\mathrm{Alq}_{3} .{ }^{23}$ It is important to note that the $1 \%$ volume concentration ratio of C540 in the S-Layer was properly chosen to ensure near $100 \%$ singlet-filtering efficiency and that no trapping states were introduced. In this experiment, it was found that $1 \%$ volume concentration ratio can satisfy the above two requirements (see supplementary material ${ }^{24}$ ). The structure of device 2 is as follows: ITO/NPB $(40 \mathrm{~nm}) / \mathrm{C} 540$ doped $\mathrm{Alq}_{3}(1 \%, 10 \mathrm{~nm}) / \mathrm{Alq}_{3}(20 \mathrm{~nm}) / \mathrm{PtOEP}$ doped $\mathrm{Alq}_{3}$ $(4 \%, 10 \mathrm{~nm}) / \mathrm{Alq}_{3}(50 \mathrm{~nm}) / \mathrm{LiF}(0.8 \mathrm{~nm}) / \mathrm{Al}(100 \mathrm{~nm})$.
Fig. 3(c) shows the change of spectrum intensity for without and with an external magnetic field $\mathrm{B}=50 \mathrm{mT}$. Figures 3(a) and 3(b) show magnified versions of the spectra around the fluorescence peak wavelength $510 \mathrm{~nm}$ and
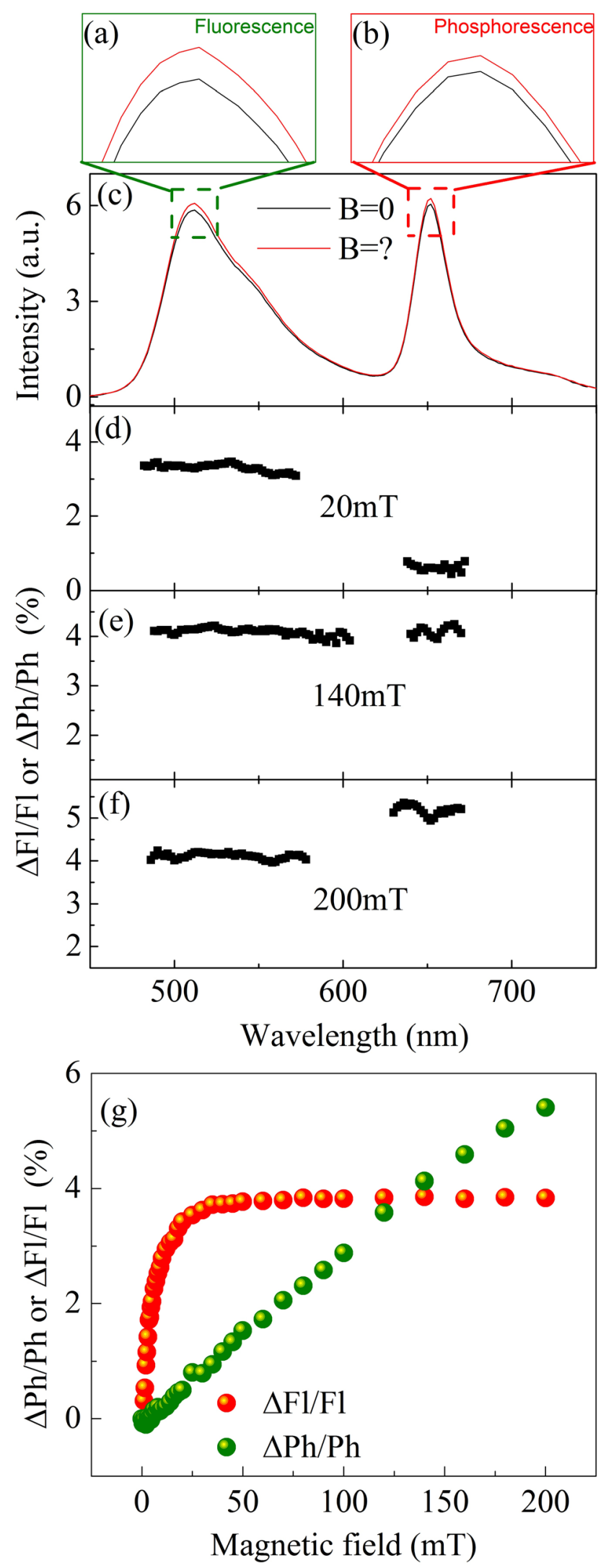

FIG. 3. Magneto-fluorescence and magneto-phosphorescence responses of device 2 . 
phosphorescence peak wavelength $650 \mathrm{~nm}$ generated by the S-Layer and T-Layer, respectively. It is important to note that the changes in fluorescence peak and phosphorescence peak due to the same external magnetic field are not the same. According to the above-mentioned definition of $\Delta \mathrm{Fl} / \mathrm{Fl}$ and $\Delta \mathrm{Ph} / \mathrm{Ph}$, Figs. 3(d) $-3(\mathrm{f})$ show the $\Delta \mathrm{Fl} / \mathrm{Fl}$ and $\Delta \mathrm{Ph} / \mathrm{Ph}$ spectra for three different external magnetic fields, namely, $\mathrm{B}=20 \mathrm{mT}, 140 \mathrm{mT}$, and $200 \mathrm{mT}$. For $\mathrm{B}=20 \mathrm{mT}, \Delta \mathrm{Fl} /$ $\mathrm{Fl}=3.5 \%$ and $\Delta \mathrm{Ph} / \mathrm{Ph}=0.6 \%$. As illustrated in Figs. 1(c) and 1(d), the magneto-fluorescence is generated by singletexciton variation, while the magneto-phosphorescence is due to triplet-exciton variation. Note that the variations of singlet and triplet excitons in the host material are simultaneously and independently monitored. Also note that the experimental results shown in Fig. 3(g) confirm that the balance between singlet and triplet excitons is changed with the application of an external magnetic field. Fig. 3(e) shows that for a 140-mT magnetic field, $\Delta \mathrm{Fl} / \mathrm{Fl}=4.0 \%$ and $\Delta \mathrm{Ph} / \mathrm{Ph}=4.1 \%$. However, when the magnetic field is further increased, the tripletexciton variation becomes higher than the singlet-exciton variation, as evident from Figs. 3(f) to 3(g).

Noticeably, the e-h pair model has been used to qualitatively explain the MEL of organic optoelectronic devices. ${ }^{25}$ According to the e-h pair model, the external magnetic field can influence the ISC between the singlet and triplet $\mathrm{e}-\mathrm{h}$ pair states, where the e-h pair is referred to the electron and hole located on different molecules and attracted to each other through Coulomb forces. Based on the e-h pair model, the change in triplet population must be equal but of opposite polarity to the change in the singlet population. However, our observed result showed that the triplet population increases quasi-linearly with the external magnetic field, whereas the e-h pair model predicts a decrease in triplet population. Therefore, to explain this behavior, other interactions occurred on excitonic levels rather than the e-h pair levels have to be considered. Sheng et al. have developed a model to quantitatively explain the impact of magnetic field on hyperfine interaction and spin-orbit coupling in organic semiconductors. ${ }^{12,13}$ However, in most OLED structures, triplet excitons, which are more sensitive to spin-orbit coupling, do not luminesce and hence cannot contribute to the MEL. This theoretical model was only indirectly verified by fitting the theoretical results with the measured organic magnetoresistance rather than the MEL. Therefore, the OLED structure (device 2) proposed in this work enables direct verification of the model through fitting the theoretical results to the measured MEL caused by the co-emission from both singlet as well as triplet exciton recombinations.

The combined Hamiltonian for the system under consideration is given by

$$
\hat{H}=\omega_{0}\left(\frac{g_{L}}{g_{S}} \hat{L}_{Z}+\hat{S}_{Z}\right)+\frac{a}{\hbar} \hat{\vec{S}} \hat{\vec{I}}+\frac{b}{\hbar} \hat{\vec{L}} \hat{\vec{S}}
$$

$\omega_{0}=\mathrm{g}_{\mathrm{s}} \mu_{\mathrm{B}} \mathrm{B} / \hbar$, where $\mu_{\mathrm{B}}$ is the electronic Bohr magneton, $\mathrm{g}_{\mathrm{L}}$ and $\mathrm{g}_{\mathrm{s}}$ are the orbital and spin $\mathrm{g}$ factors, respectively, $\overrightarrow{\mathrm{L}}$ and $\vec{S}$ are the electronic orbital and spin angular momentums, respectively, $\overrightarrow{\mathrm{I}}$ is the proton spin, $\mathrm{a}$ and $\mathrm{b}$ are the hyperfine coupling constant and spin-orbit interaction strength, respectively.
Using Eq. (1), and following the analysis reported in Refs. 12 and 13, yields:

$$
\frac{\Delta \mathrm{EL}}{\mathrm{EL}}=\alpha \frac{\mathrm{B}^{2}}{\mathrm{~B}^{2}+\mathrm{B}_{0}^{2}}+\beta \frac{\mathrm{B}^{2}}{\left(|\mathrm{~B}|+\mathrm{B}_{1}\right)^{2}} .
$$

$\mathrm{B}_{0}$ and $\mathrm{B}_{1}$ are parameters related to the hyperfine interaction scale and the spin-orbit coupling scale, respectively. From Eq. (2), one can see that, in principle, both components, namely, the hyperfine interaction and spin-orbit coupling, could contribute to the MEL. Figure 4 shows experimental theoretical fits for magnetophosphorescence and magnetofluorescence using the two-mechanism model of Eq. (1) and the expression in Eq. (2). Excellent agreement between experimental and theoretical data is observed. The measured magneto-phosphorescence of device 1 reveals that the MEL consists of two components, namely (i) a relatively steep component due to the hyperfine interaction, which is dominant at low magnetic fields and (ii) a component due to spinorbit coupling, which is dominant at high magnetic fields. Fitting the experimental data with Eq. (2) yields $B_{0}=4.5 \mathrm{mT}$ and $\mathrm{B}_{1}=75 \mathrm{mT}$, which are consistent with the fitted values for OLEDs. ${ }^{12,13,19,26}$ The first term and second term in Eq. (2) are crucial for predicting the singlet-exciton variation and triplet-exciton variation in external magnetic fields shown in Fig. 3(g). For device 2, as shown in Fig. 4, the red line and the blue line through the data points are fits using the hyperfine interaction term $\frac{\mathrm{B}^{2}}{\mathrm{~B}^{2}+\mathrm{B}_{0}^{2}}$ and the spin-orbit coupling term $\frac{B^{2}}{\left(|B|+B_{1}\right)^{2}}$, respectively. For this device, the estimated values of $\mathrm{B}_{0}$ and $\mathrm{B}_{1}$ are $4.5 \mathrm{mT}$ and $82 \mathrm{mT}$, respectively, which are almost the same as those for device 1. This confirms that the MEL components for device 1 originate from hyperfine-interaction-dominated singlet variation and spin-orbit-coupling-dominated triplet variation.

Finally, although the singlet-exciton and triplet exciton variations can be well described by the theory based on the classic model that considers hyperfine interaction and spinorbit coupling simultaneously, the details of the spin

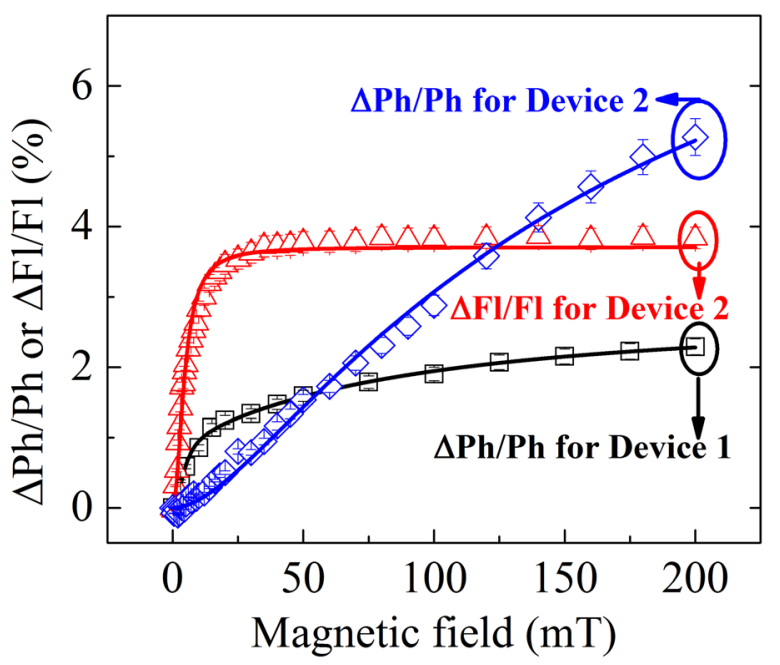

FIG. 4. Experimental and theoretical fits for magnetophosphorescence and magneto-fluorescence. The black, red, and blue lines through the measured data points are fits using the two-mechanism model, hyperfine interaction model, and spin-orbit coupling model, respectively. 
dynamics, regarding how the hyperfine interaction and the spin-orbit coupling influence the singlet variation and triplet variation, are still unclear. This requires advanced modeling taking into account quantum correlation. Recently, Si et al. have discussed the quenching of the quantum correlation between the carrier's spin and its local environment in the presence of an external magnetic field. ${ }^{27}$ According to their theory, applying an external magnetic field can increase the possibility of carrier delocalization in organic semiconductors, and many parameters are linked to the delocalization process such as hopping rate, and charge detrapping through the triplet exciton-charge interaction. For example, if the hopping rates of free electrons and holes increase, the encountering probability between the electrons and holes is increased accordingly. Therefore, as predicted from the theory, both the singlet exciton formation rate and triplet exciton formation rate increases by applying an external magnetic field, which is consistent with our observation.

In conclusion, we have proposed and developed an OLED structure employing a singlet-exciton filtering and detection layer in conjunction with a triplet-exciton detection layer, allowing simultaneous observation of singlet and tripletexciton variations in an external magnetic field. Experimental results have confirmed that the magnetoelectroluminescence of OLEDs driven by external magnetic field comprises the Lorentzian singlet variation term and a non-Lorentzian triplet variation term. Such experimental results will help researchers to better understand the spin dynamics of organic semiconductors. In addition, the dependence of singlet-exciton variation on the magnetic field is different from that of the tripletexciton variation, thus, it is possible to manipulate the electroluminescent emission colors of the proposed OLED structure through an external magnetic field. The observed quasi-linear non-Lorentzian triplet variation is especially useful for the development of future organic magnetic-field sensors.

This work research is supported by Edith Cowan University, the Department of Industry, Innovation, Science, Research, and Tertiary Education, Australia.

${ }^{1}$ C. W. Tang and S. A. Vanslyke, Appl. Phys. Lett. 51, 913-915 (1987).

${ }^{2}$ B. F. Ding, Y. Q. Zhan, Z. Y. Sun, X. M. Ding, X. Y. Hou, Y. Z. Wu, I. Bergenti, and V. Dediu, Appl. Phys. Lett. 93, 183307 (2008).
${ }^{3}$ B.-F. Ding, X.-Y. Hou, and K. Alameh, Appl. Phys. Lett. 101, 133305 (2012).

${ }^{4}$ X. Li, W. C. H. Choy, L. Huo, F. Xie, W. E. I. Sha, B. Ding, X. Guo, Y. Li, J. Hou, J. You, and Y. Yang, Adv. Mater. 24, 3046-3052 (2012).

${ }^{5}$ F. Garnier, R. Hajlaoui, A. Yassar, and P. Srivastava, Science 265, 1684-1686 (1994).

${ }^{6}$ M. A. Baldo, D. F. O’Brien, Y. You, A. Shoustikov, S. Sibley, M. E. Thompson, and S. R. Forrest, Nature 395, 151-154 (1998).

${ }^{7}$ Z. H. Xiong, D. Wu, Z. V. Vardeny, and J. Shi, Nature 427, 821-824 (2004).

${ }^{8}$ V. A. Dediu, L. E. Hueso, I. Bergenti, and C. Taliani, Nat. Mater. 8, 707-716 (2009).

${ }^{9}$ A. J. Drew, J. Hoppler, L. Schulz, F. L. Pratt, P. Desai, P. Shakya, T. Kreouzis, W. P. Gillin, A. Suter, N. A. Morley, V. K. Malik, A. Dubroka, K. W. Kim, H. Bouyanfif, F. Bourqui, C. Bernhard, R. Scheuermann, G. J. Nieuwenhuys, T. Prokscha, and E. Morenzoni, Nat. Mater. 8, 109-114 (2009).

${ }^{10}$ B. Hu and Y. Wu, Nat. Mater. 6, 985-991 (2007).

${ }^{11}$ B. Ding, Q. Song, and K. Alameh, Appl. Phys. Lett. 104, 203302 (2014).

${ }^{12}$ Y. Sheng, T. D. Nguyen, G. Veeraraghavan, Ö. Mermer, and M. Wohlgenannt, Phys. Rev. B 75, 035202 (2007).

${ }^{13}$ Y. Sheng, T. D. Nguyen, G. Veeraraghavan, O. Mermer, M. Wohlgenannt, S. Qiu, and U. Scherf, Phys. Rev. B 74, 045213 (2006).

${ }^{14}$ B. Ding, Y. Yao, X. Sun, X. Gao, Z. Xie, Z. Sun, Z. Wang, X. Ding, Y. Wu, X. Jin, W. C. H. Choy, C.-Q. Wu, and X. Hou, Phys. Rev. B 82, 205209 (2010).

${ }^{15}$ M. Nishioka, Y.-B. Lee, A. M. Goldman, Y. Xia, and C. D. Frisbie, Appl. Phys. Lett. 91, 092117 (2007).

${ }^{16}$ A. Buchschuster, T. D. Schmidt, and W. Brütting, Appl. Phys. Lett. 100, 123302 (2012).

${ }^{17}$ A. Riminucci, M. Prezioso, C. Pernechele, P. Graziosi, I. Bergenti, R. Cecchini, M. Calbucci, M. Solzi, and V. Alek Dediu, Appl. Phys. Lett. 102, 092407 (2013).

${ }^{18}$ Y. Zhang, R. Liu, Y. L. Lei, and Z. H. Xiong, Appl. Phys. Lett. 94, 083307 (2009).

${ }^{19}$ P. A. Bobbert, T. D. Nguyen, F. W. A. van Oost, B. Koopmans, and M. Wohlgenannt, Phys. Rev. Lett. 99, 216801 (2007).

${ }^{20}$ P. Chen, Q. L. Song, W. C. H. Choy, B. F. Ding, Y. L. Liu, and Z. H. Xiong, Appl. Phys. Lett. 99, 143305 (2011).

${ }^{21}$ M. Reufer, M. J. Walter, P. G. Lagoudakis, B. Hummel, J. S. Kolb, H. G. Roskos, U. Scherf, and J. M. Lupton, Nat. Mater. 4, 340-346 (2005).

${ }^{22}$ J. M. Lupton and C. Boehme, Nat. Mater. 7, 598 (2008).

${ }^{23}$ Y. Wu, H. R. Wu, Y. C. Zhou, Y. Q. Zhan, J. Zhou, X. M. Ding, and X. Y. Hou, Appl. Phys. Lett. 88, 123512 (2006).

${ }^{24}$ See supplementary material at http://dx.doi.org/10.1063/1.4890315 for additional experimental details on the relationship between the doping ratio and trapping states.

${ }^{25}$ W. Qin, K. Gao, S. Yin, and S. J. Xie, J. Appl. Phys. 113, 193901 (2013).

${ }^{26}$ T. D. Nguyen, G. Hukic-Markosian, F. Wang, L. Wojcik, X.-G. Li, E. Ehrenfreund, and Z. V. Vardeny, Nat. Mater. 9, 345-352 (2010).

${ }^{27}$ W. Si, Y. Yao, X. Hou, and C.-Q. Wu, Org. Electron. 15, 824-828 (2014). 